\title{
Identification of complications that have a significant effect on length of stay after spine surgery and predictive value of 90-day readmission rate
}

\author{
Sanjay Yadla, MD, ${ }^{1}$ George M. Ghobrial, MD,, Peter G. Campbell, MD, ${ }^{1}$ Mitchell G. Maltenfort, PhD, ${ }^{2}$ \\ James S. Harrop, MD, ${ }^{1}$ John K. Ratliff, MD, ${ }^{3}$ and Ashwini D. Sharan, MD1 \\ Departments of ${ }^{1}$ Neurological Surgery and ${ }^{2}$ Orthopedic Surgery, Thomas Jefferson University, Philadelphia, Pennsylvania; and \\ ${ }^{3}$ Department of Neurological Surgery, Stanford University, Palo Alto, California
}

\begin{abstract}
OBJECT Complications after spine surgery have an impact on overall outcome and health care expenditures. The increased cost of complications is due in part to associated prolonged hospital stays. The authors propose that certain complications have a greater impact on length of stay (LOS) than others and that those complications should be the focus of future targeted prevention efforts. They conducted a retrospective analysis of a prospectively maintained database to identify complications with the greatest impact on LOS as well as the predictive value of these complications with respect to 90-day readmission rates.
\end{abstract}

METHODS Data on 249 patients undergoing spine surgery at Thomas Jefferson University from May to December 2008 were collected by a study auditor. Any complications occurring within 30 days of surgery were recorded as was overall LOS for each patient. Stepwise regression analysis was performed to determine whether specific complications had a statistically significant effect on LOS. For correlation, all readmissions within 90 days were recorded and organized by complication for comparison with those complications affecting LOS.

RESULTS The mean LOS for patients without postoperative complications was 6.9 days. Patients who developed pulmonary complications had an associated increase in LOS of 11.1 days $(p<0.005)$. The development of a urinary tract infection (UTI) was associated with an increase in LOS of 3.4 days $(p=0.002)$. A new neurological deficit was associated with an increase in LOS of 8.2 days $(p=0.004)$. Complications requiring return to the operating room $(O R)$ showed a trend toward an increase in LOS of 4.7 days $(p=0.09)$, as did deep wound infections ( 3.3 days, $p=0.08)$. The most common reason for readmission was for wound drainage $(n=21$; surgical drainage was required in 10 [4.01\%] of these 21 cases). The most common diagnoses for readmission, in decreasing order of incidence, were categorized as hardware malpositioning $(n=4)$, fever $(n=4)$, pulmonary $(n=2)$, UTI $(n=2)$, and neurological deficit $(n=1)$. Complications affecting LOS were not found to be predictive of readmission $(p=0.029)$.

CONCLUSIONS Postoperative complications in patients who have undergone spine surgery are not uncommon and are associated with prolonged hospital stays. In the current cohort, the occurrence of pulmonary complications, UTI, and new neurological deficit had the greatest effect on overall LOS. Further study is required to determine the causative factors affecting readmission. These specific complications may be high-yield targets for cost reduction and/or prevention efforts. http://thejns.org/doi/abs/10.3171/2015.3.SPINE14318

KEY WORDS hospital stay; length of stay; complications; adverse events; health care expenditures; health care costs

$\mathrm{A}$ CCURATE analyses of the costs associated with spine surgery are increasingly necessary as the demonstration of benefit and cost-effectiveness of surgical interventions becomes more important to health care payers and government. ${ }^{4,21}$ Complications are associated with increased costs after spine surgery due to the additional services required to mitigate them. ${ }^{21}$ These additional services often require longer hospital stays and occasionally readmission or return to the operating room (OR)..$^{1,20,23}$

Multiple studies have investigated the relationship between prolonged hospital stay and costs in several arenas including general surgery, urological procedures, and cardiac interventions. ${ }^{12,13,14,17}$ These reports have demonstrated the direct relationship between length of stay (LOS) and increasing expenditures. We hypothesized that certain

ABBREVIATIONS BMI = body mass index; DVT = deep venous thrombosis; EMR = electronic medical record; LOS = length of stay; OR = operating room; UTI = urinary tract infection.

SUBMITTED March 25, 2014. ACCEPTED March 25, 2015

INCLUDE WHEN CITING Published online August 28, 2015; DOI: 10.3171/2015.3.SPINE14318. 
complications have a greater impact on LOS than others. Identification of these specific complications might provide a rationale for focused prevention efforts by health care providers in the future. To test this hypothesis, we retrospectively analyzed a prospectively maintained database of patients who underwent spine surgery at our institution over a 7-month period.

\section{Methods}

All patients who underwent spine surgery by the neurosurgical service at the Thomas Jefferson University Hospital from May to December 2008 were eligible for inclusion and consecutively enrolled. There were no exclusion criteria for this study, and its design was purely observational. Data on age, sex, preoperative comorbidity, underlying pathology, surgical approach and technique, complications, and LOS were entered prospectively into a central database by a study auditor. Any complications occurring during the initial hospital stay and the first 30 postoperative days were recorded. Independently, all readmissions for the same patient population and their diagnosis on readmission for a period of 90 days were recorded. All patients were followed at the Department of Neurological Surgery outpatient clinics for 90 days. An independent study auditor from the neurosurgical department nursing staff was designated to evaluate postoperative patients in the inpatient and outpatient clinic and determine if any complications had occurred. Patients were excluded from the trial if they declined to participate or if the study auditor was not available to enroll them at presentation (i.e., weekends, holidays). The protocol was approved by the local institutional review board prior to commencement.

Complications were defined as adverse events leading to further interventions, return to the OR, or permanent sequelae. They were classified by category into pulmonary (i.e., pneumonia, pulmonary embolism), cardiac (i.e., myocardial infarction, supraventricular tachycardia), urinary tract infection (UTI), deep wound infection, return to the OR (i.e., for hardware malposition or migration, epidural hematoma, cerebrospinal fluid leak), new neurological deficits, and deep venous thrombosis (DVT). They were recorded as binomial variables (yes or no) based upon their presence or absence during a patient's postoperative course. LOS was recorded as a continuous variable in whole days. Complications during the inpatient period, readmissions, and transfers were available for data collection on inpatient electronic medical records (EMRs) and paper charts. For the outpatient setting, EMRs with integrated access to patient phone calls, dictations, radiology reports, outpatient providers, and laboratory information were available for complications reporting. If a phone call was made regarding a suspected adverse event, an inquiry was made by the auditor for neurosurgical staff to provide follow-up and assess if an adverse event had occurred.

A linear regression model using all complication categories was constructed. LOS followed a negative binomial distribution, so statistical analysis was performed using an appropriate statistical model in $\mathrm{R}$ version 2.15.0 (R Foundation for Statistical Computing; www.r-project. org). The negative binomial model was implemented using the "glm.nb" function in the R package "MASS" and with the link function "identity" so that the returned parameter estimates were all additive terms. Backward stepwise regression methods were then used to create a parsimonious model where only the parameters that were associated with the accuracy of the model were included. The 95\% confidence interval for each estimate was calculated. A p value of less than 0.05 was considered statistically significant.

\section{Results}

The mean age of the 249 patients included in the study was 55.1 years. There were 115 female and 134 male patients in the series. The majority of patients underwent surgery for degenerative disease (161). Thirty-nine patients underwent surgery for trauma, 29 for neoplasm, and 20 for infectious pathologies. The mean body mass index (BMI) was $29.6 \mathrm{~kg} / \mathrm{m}^{2}$ (Table 1).

One-hundred seventeen patients (47\%) had no complications postoperatively. One-hundred thirty-two patients (53\%) had at least 1 complication. The number of complications experienced by patients ranged from 1 to 7 , with a mean of 1.8 complications per patient who experienced at least 1 . The mean LOS for patients without a complicated postoperative course was 6.9 days; the mean LOS for those with a complication was 13.5 days ( $p<0.05$; Fig. 1). Eighteen patients developed pulmonary complications, 12 developed cardiac complications, 33 experienced UTI, 28 had deep wound infections, 13 were required to return to the OR, 9 developed a new neurological deficits, and 17 developed DVT (Fig. 2).

Regression analysis showed that the occurrence of a pulmonary complication, a UTI, or a new neurological deficit increased the LOS by 11.1 days, 3.4 days, and 9.1 days, respectively ( $\mathrm{p}<0.05$; Table 2 ). A return to the OR showed a trend toward an associated increase in LOS of 4.7 days $(\mathrm{p}=0.09)$; deep wound infections also showed a trend toward an associated increase in LOS of 3.3 days ( $\mathrm{p}$ $=0.08)$.

TABLE 1. Summary of cohort characteristics*

\begin{tabular}{cc}
\hline Characteristic & Value \\
\hline Mean age, yrs & 55.1 \\
\hline Mean BMl, $\mathrm{kg} / \mathrm{m}^{2}$ & 29.6 \\
\hline Sex, $\mathrm{n}(\%)$ & $134(54)$ \\
\hline $\mathrm{M}$ & $115(46)$ \\
\hline $\mathrm{F}$ & \\
\hline Pathology & $161(64.7)$ \\
\hline Degenerative & $39(15.7)$ \\
\hline Trauma & $29(11.6)$ \\
\hline Tumor & $20(8)$ \\
\hline Infection & $205(82)$ \\
\hline Instrumentation & $120(48.2)$ \\
\hline Level of pathology & $3(1.2)$ \\
\hline Cervical & $126(50.6)$ \\
\hline Cervicothoracic &
\end{tabular}

* Values are number of patients (\%) except where otherwise indicated. 


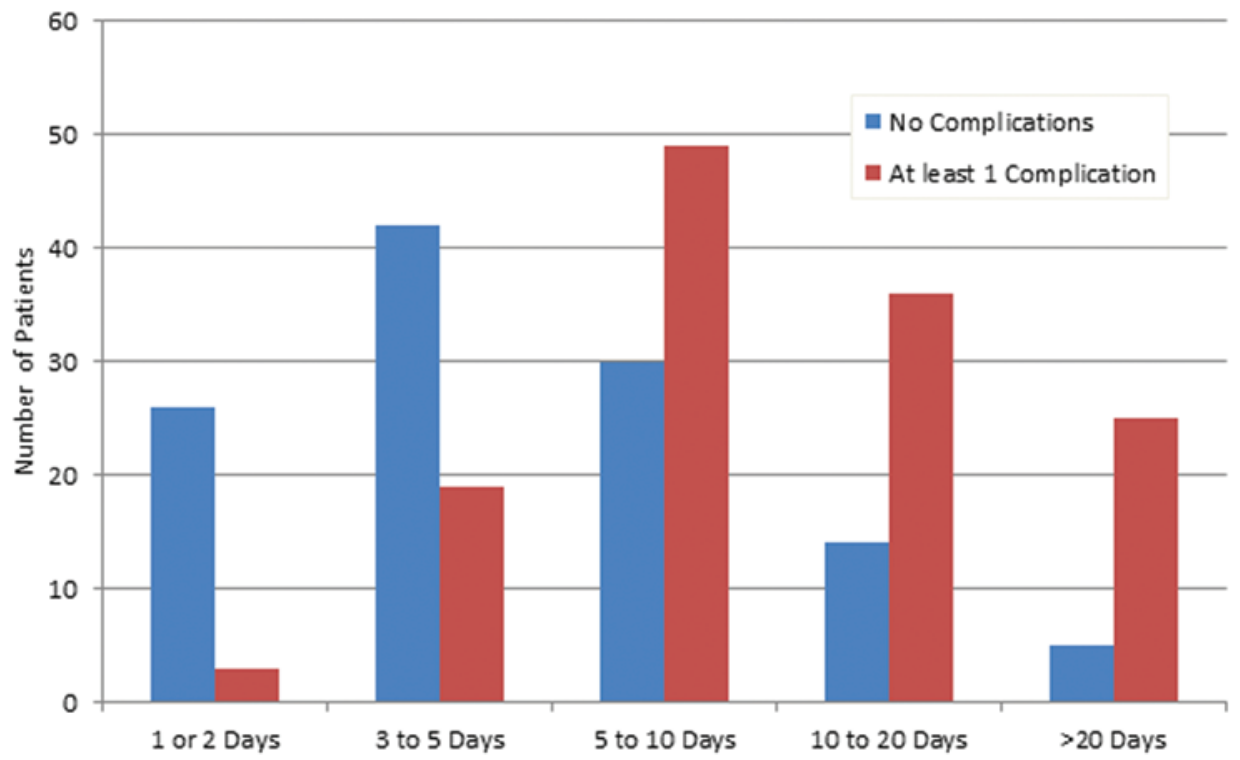

FIG. 1. Bar graph showing the distribution of LOS for all patients included in the study. Figure is available in color online only.

Readmissions were recorded for a 90-day period (Fig. 3 ), during which 37 readmissions (14.9\%) occurred. The most common indication for readmission was for wound drainage $(n=21)$, 10 of which underwent surgical drainage $(4.01 \%)$. The most common diagnoses for readmission in decreasing order of incidence were categorized as hardware malpositioning $(n=4)$, fever $(n=4)$, pulmonary $(n=$ $2)$, UTI $(\mathrm{n}=2)$, and neurological deficit $(\mathrm{n}=1)$. Complications affecting LOS were not found to be predictive of readmission $(\mathrm{p}=0.029)$.

\section{Discussion}

The current analysis found that the occurrence of a complication is associated with an increased LOS. Specific complications were found to have a statistically significant increase in the LOS. These included pulmonary complications, which increased the LOS by 11.1 days; UTI, which increased the LOS by 3.4 days; and new neurological defi- cits, which increased the LOS by 9.1 days. An increased LOS was associated with an increase in health care expenditures for the admission in this cohort as reported in previous publications..$^{20,21}$ As a cost-containment measure, the value in predicting readmission risk can be beneficial. In this analysis, readmissions over 90 days did not correlate with the common adverse events prolonging LOS. This is an interesting finding not previously addressed, as one would expect problematic adverse events that prolong LOS to more commonly result in readmission.

In this study, pulmonary complications had the greatest impact on additional LOS. Winslow et al. reported that the incidence of pulmonary complications during admission for acute cervical spinal cord injury was directly related to cost and LOS, and that these were a better predictor of hospital costs than the level of injury. ${ }^{22}$ Khan et al. investigated the association between hospital costs and LOS in 7457 patient undergoing noncardiac surgery at a tertiary care

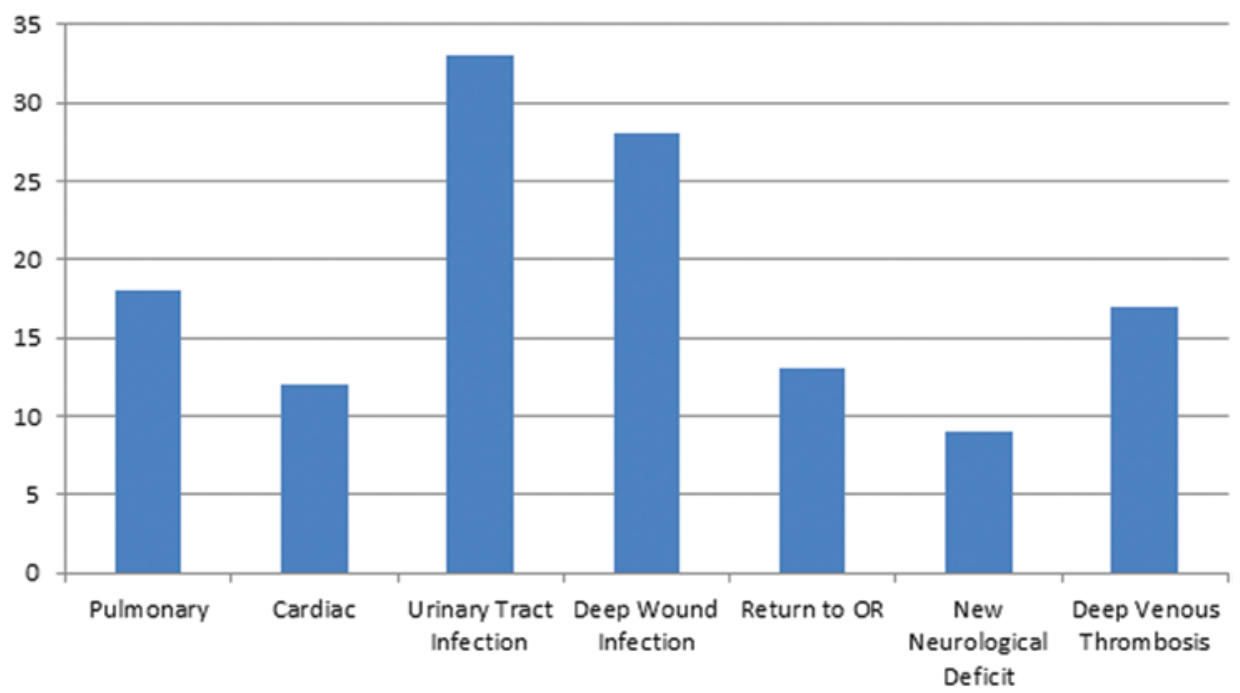

FIG. 2. Bar graph showing the distribution of complications in the current cohort. Figure is available in color online only. 
TABLE 2. Summary of results of regression analysis

\begin{tabular}{lccl}
\hline Complication & $\begin{array}{c}\text { Mean Increase } \\
\text { in LOS (days) }\end{array}$ & $\begin{array}{c}95 \% \mathrm{Cl} \\
\text { (days) }\end{array}$ & $\begin{array}{c}\mathrm{p} \\
\text { Value* }\end{array}$ \\
\hline Pulmonary & 11.1 & 3.3 to 8.9 & $\mathbf{0 . 0 0 5}$ \\
\hline Cardiac & 4.8 & -2.2 to 11.8 & 0.11 \\
\hline UTI & 3.4 & -0.2 to 7.0 & $\mathbf{0 . 0 0 2}$ \\
\hline $\begin{array}{l}\text { Deep wound } \\
\text { infection }\end{array}$ & 3.3 & -0.3 to 6.9 & 0.08 \\
\hline $\begin{array}{l}\text { Return to OR } \\
\text { New neurological } \\
\text { deficit }\end{array}$ & 4.7 & -2.5 to 11.9 & 0.09 \\
\hline DVT† & 9.1 & -0.7 to 18.9 & $\mathbf{0 . 0 0 4}$ \\
\hline
\end{tabular}

* Statistically significant values are shown in bold type.

$\dagger$ Not included in final model.

center. ${ }^{11}$ They found that the occurrence of a complication increased costs by $78 \%$ and LOS by $114 \%$ and that pneumonia specifically was associated with an $89 \%$ increase in LOS. Several studies have looked at the institution of preoperative pulmonary physiotherapy, perioperative noninvasive ventilation, and other interventions for the prevention of postoperative pulmonary complications. ${ }^{3,7,9,10} \mathrm{~A}$ number of studies have focused on DVT prophylaxis for the prevention of postoperative pulmonary embolism in traumatic and nontraumatic spine patients. ${ }^{2,15,16,18,19}$

Patients who have undergone spine surgery may be prone to UTIs due to periods of immobilization and the need for indwelling bladder catheters in the postoperative period. Although several studies have investigated the effectiveness of UTI prevention methods in the general surgical population, there are few studies specific to patients who underwent spine surgery ${ }^{6,8}$ This may be an area where further studies are warranted. It is surprising that UTI impacted LOS to a greater extent than did wound infection (Table 2) in our study. The authors feel this was due to an overdiagnosis of UTI, resulting in the overuse of intravenous antibiotics for presumed infection. This presumed overdiagnosis was accounted for by older practices of UTI diagnosis and treatment seen in 2008. In the perioperative period of this study, it was commonplace to treat urinalysis finding of high white cells or leukocyte esterase and nitrite positive as evidence of UTI. Intravenous antibiotic therapy was initiated prior to culture results and continued even if the results were negative. This was due to concern for limiting bacteremia in the perioperative setting, especially in the case of instrumentation.

The incidence of complications in the current cohort is higher than many previous reports. We believe that there are several reasons for this observation including the prospective nature of evaluation, the application of a liberal definition of complication, and the complicated nature of patients' underlying pathologies. A large proportion of patients underwent instrumentation procedures, which have been associated with an increased incidence of complications as well. ${ }^{5}$

A limitation of the current study is that complications such as deep wound infections may surface or lead to readmission beyond 30 postoperative days. This might lead to an underestimate of their true impact on health expenditures using the current methodology. Another limitation is that many complications are interrelated or due to the same underlying pathology making it difficult to isolate their unique effects. For instance, a patient immobilized by a high cervical injury classified as Grade A on the American Spinal Injury Association Impairment Scale would be more likely to develop pulmonary complications as well as DVT or UTI. Furthermore, instances of patients seen and evaluated in a community center for a postsurgical complication may go underreported if that facility does not seek transfer or relay their findings to our outpatient staff. These events are difficult to fully track and are thought to be very infrequent.

There were several limitations regarding the tracking of hospital LOS. Table 2 lists the factors affecting hospital LOS that were able to be objectively assessed. However, there were a number of factors not captured by this study,

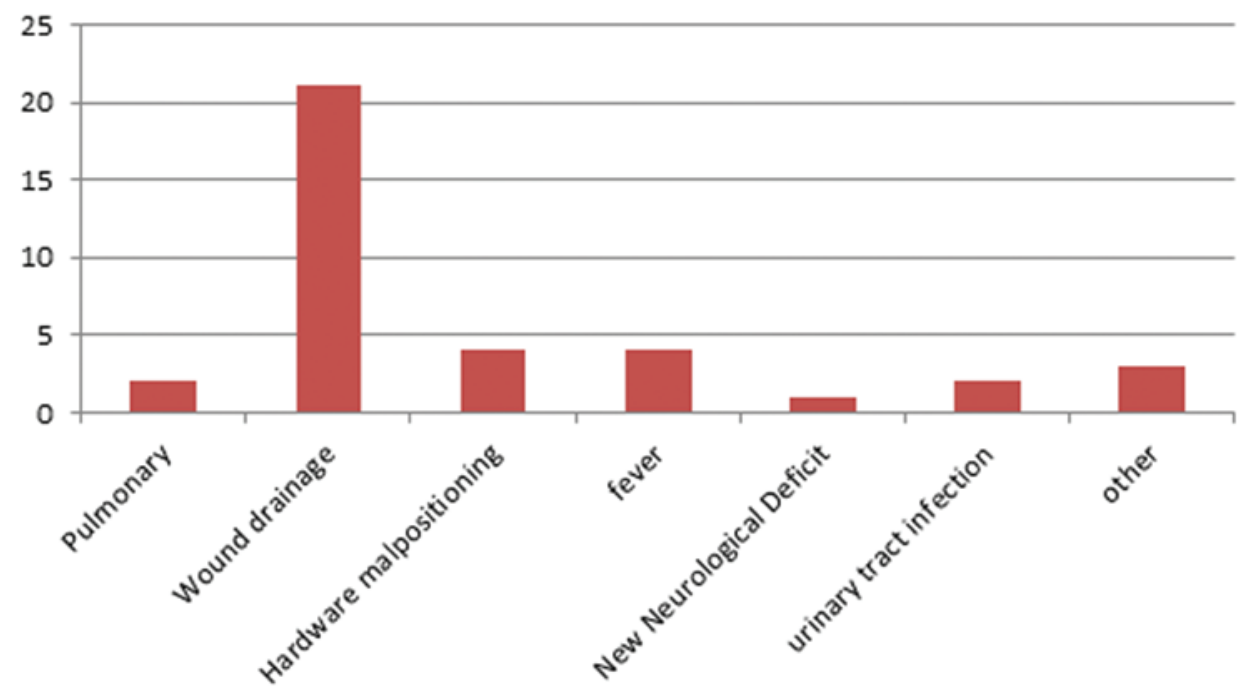

FIG. 3. Bar graph showing the distribution of complications affecting readmission in the current cohort. Figure is available in color online only. 
such as socioeconomic factors, unavailable to the staff in a way that could be reliably tracked. One example would be a patient unable to obtain personal transportation home resulting in a delayed discharge. Another example would be an uninsured patient requiring additional time to obtain medical assistance for rehabilitation placement, home physical therapy, or infusion services, all of which prolong LOS. Anecdotally, patients with chronic pain requiring prolonged intravenous analgesia or the assistance of an inpatient consultant specializing in pain management have prolonged LOS, but pain was not tracked in this study.

\section{Conclusions}

We have identified, through the retrospective analysis of a prospectively maintained database, that pulmonary complications, urinary tract infections, and new neurological deficits had a statistically significant effect on LOS, and reoperation and wound infection had a trend towards a statistically significant effect on LOS in the current cohort. The resulting increased LOS is likely to lead to a direct increase in health care expenditures for a given patient, and these complications would, therefore, be attractive targets for complication-prevention programs in patients who have undergone spine surgery.

\section{References}

1. Acosta FL, Cloyd JM, Aryan HE, Ames CP: Perioperative complications and clinical outcomes of multilevel circumferential lumbar spinal fusion in the elderly. J Clin Neurosci 16:69-73, 2009

2. Baaj AA, Gokaslan ZL: Venous thromboembolism in spine surgery. World Neurosurg 78:433, 2012

3. Bagan P, Bouayad M, Benabdesselam A, Landais A, Mentec $\mathrm{H}$, Couffinhal JC: Prevention of pulmonary complications after aortic surgery: evaluation of prophylactic noninvasive perioperative ventilation. Ann Vasc Surg 25:920-922, 2011

4. Campbell PG, Malone J, Yadla S, Chitale R, Nasser R, Maltenfort MG, et al: Comparison of ICD-9-based, retrospective, and prospective assessments of perioperative complications: assessment of accuracy in reporting. J Neurosurg Spine 14:16-22, 2011

5. Campbell PG, Yadla S, Malone J, Maltenfort MG, Harrop JS, Sharan AD, et al: Complications related to instrumentation in spine surgery: a prospective analysis. Neurosurg Focus 31(4):E10, 2011

6. Chan YM, Ngai SW, Hon E, So WK: Could the incidence of postoperative urinary tract infection be reduced by reversing the sequence of vaginal cleansing and urethral catheterization? J Hosp Infect 46:67-72, 2000

7. Cook MW, Lisco SJ: Prevention of postoperative pulmonary complications. Int Anesthesiol Clin 47:65-88, 2009

8. Garibaldi RA: Postoperative pneumonia and urinary-tract infection: epidemiology and prevention. J Hosp Infect 11 (Suppl A):265-272, 1988

9. Hayden SP, Mayer ME, Stoller JK: Postoperative pulmonary complications: risk assessment, prevention, and treatment. Cleve Clin J Med 62:401-407, 1995

10. Inoue J, Ono R, Makiura D, Kashiwa-Motoyama M, Miura Y, Usami M, et al: Prevention of postoperative pulmonary complications through intensive preoperative respiratory rehabilitation in patients with esophageal cancer. Dis Esophagus 26: 68-75, 2013

11. Khan NA, Quan H, Bugar JM, Lemaire JB, Brant R, Ghali WA: Association of postoperative complications with hos- pital costs and length of stay in a tertiary care center. J Gen Intern Med 21:177-180, 2006

12. Kim SP, Shah ND, Karnes RJ, Weight CJ, Frank I, Moriarty JP, et al: The implications of hospital acquired adverse events on mortality, length of stay and costs for patients undergoing radical cystectomy for bladder cancer. J Urol 187:2011-2017, 2012

13. McAleese P, Odling-Smee W: The effect of complications on length of stay. Ann Surg 220:740-744, 1994

14. Nuis RJ, Piazza N, Van Mieghem NM, Otten AM, Tzikas A, Schultz CJ, et al: In-hospital complications after transcatheter aortic valve implantation revisited according to the Valve Academic Research Consortium definitions. Catheter Cardiovasc Interv 78:457-467, 2011

15. Paciaroni M, Ageno W, Agnelli G: Prevention of venous thromboembolism after acute spinal cord injury with lowdose heparin or low-molecular-weight heparin. Thromb Haemost 99:978-980, 2008

16. Richard H III, Widlus DM, Scalea TM: Inferior vena cava filters in trauma: balancing pulmonary embolism prevention with the risk of deep venous thrombosis. J Trauma 69:1003-1004, 2010

17. Rubinstein E, Green M, Modan M, Amit P, Bernstein L, Rubinstein A: The effects of nosocomial infections on the length and costs of hospital stay. J Antimicrob Chemother 9 (Suppl A):93-100, 1982

18. Takahashi H, Yokoyama Y, Iida Y, Terashima F, Hasegawa $\mathrm{K}$, Saito T, et al: Incidence of venous thromboembolism after spine surgery. J Orthop Sci 17:114-117, 2012

19. Teasell RW, Hsieh JT, Aubut JA, Eng JJ, Krassioukov A, Tu L: Venous thromboembolism after spinal cord injury. Arch Phys Med Rehabil 90:232-245, 2009

20. Teufack SG, Campbell P, Jabbour P, Maltenfort M, Evans J, Ratliff JK: Potential financial impact of restriction in "never event" and periprocedural hospital-acquired condition reimbursement at a tertiary neurosurgical center: a single-institution prospective study. J Neurosurg 112:249-256, 2010

21. Whitmore RG, Stephen J, Stein SC, Campbell PG, Yadla S, Harrop JS, et al: Patient comorbidities and complications following spinal surgery: a societal-based cost analysis. Spine (Phila Pa 1976) 37:1065-1071, 2012

22. Winslow C, Bode RK, Felton D, Chen D, Meyer PR Jr: Impact of respiratory complications on length of stay and hospital costs in acute cervical spine injury. Chest 121:1548-1554, 2002

23. Yadla S, Malone J, Campbell PG, Maltenfort MG, Harrop JS, Sharan AD, et al: Early complications in spine surgery and relation to preoperative diagnosis: a single-center prospective study. J Neurosurg Spine 13:360-366, 2010

\section{Disclosure}

Dr. Harrop reports being a consultant for DePuy Spine and receiving clinical or research support for the study described (includes equipment and material) from Tejin and Bioventus.

\section{Author Contributions}

Conception and design: Yadla, Ratliff, Sharan. Acquisition of data: Yadla, Campbell, Sharan. Analysis and interpretation of data: Campbell, Maltenfort. Drafting the article: Sharan. Critically revising the article: Ghobrial, Harrop, Ratliff.

Reviewed submitted version of manuscript: Ghobrial, Harrop. Study supervision: Harrop.

\section{Correspondence}

Sanjay Yadla, Department of Neurosurgery, Thomas Jefferson University, 909 Walnut St., 2nd Fl., Philadelphia, PA 19107. email: sanjayadla@gmail.com. 